\title{
ENSAIO DE PULLOUT EM MATRIX DE POLIÉSTER COM FIBRA DE PALF*
}

\section{Resumo}

Gabriel Oliveira Glória' Maria Carolina Andrade Teles ${ }^{1}$ Felipe Perissé Duarte Lopes ${ }^{2}$ Maycon de Almeida Gomes ${ }^{3}$ Frederico Muylaert Margem ${ }^{4}$ Carlos Maurício Fontes Vieira ${ }^{5}$ Sérgio Neves Monteiro ${ }^{6}$ Jean Igor Margem ${ }^{7}$

A sociedade cada vez mais está buscando por materiais ecologicamente corretos em detrimento de materiais nocivos ao meio ambiente. Nesse novo contexto as fibras naturais estão, cada vez mais substituindo as fibras sintéticas, por exemplo, a fibra de vidro. Além das vantagens ambientais, as fibras naturais são mais baratas do que os sintéticos. O ensaio de pullout estuda a adesão da fibra na matriz de poliéster e a interação das fibras com a matriz de poliéster, também chamados de biocompósitos. Portanto, o presente estudo analisou a fibra de PALF por meio de ensaio de pullout. $O$ ensaio verificou que o comprimento de embutimento crítico da fibra de PALF em matriz de poliéster é de $1,20 \mathrm{~mm}$ e a tensão cisalhante na interface fibra/matriz é de 11,66 Mpa.

Palavras-chave: Fibras de PALF; Ensaio de Pullout; Materiais Compósitos; Fibras Naturais.

\section{PULLOUT TESTS IN POLYESTER MATRIX COMPOSITES WITH PALF FIBER}

\section{Abstract}

The society is more and more looking for environmentally friendly materials at contrasting with harmful materials to the environment. In this new context, natural fibers are replacing synthetic fibers, for example, fiberglass. In addition to environmental advantages, natural fibers are cheaper than synthetics. The pullout test studies the adhesion of the fiber in the polyester matrix and the interaction of fibers with the polyester matrix, also called biocomposites. Therefore, the present study analyzed the PALF fiber by means of the pullout test. The tests shows that the critical inlay length of the PALF fiber in the polyester matrix is $1.20 \mathrm{~mm}$ and the shear stress at the fiber / matrix interface is $11.66 \mathrm{MPa}$.

Keywords: PALF fibers; Pullout test; Composites; Natural Fibers.

1 Graduando(a) em Engenharia Metalúrgica e de Materiais, bolsista de iniciação científica, LAMAV, UENF, Campos dos Goytacazes, Rio de Janeiro - Brasil.

2 Engenheiro Metalúrgico, Doutor em Ciência e Engenharia de Materiais, Pós-Doutorando, LAMAV, UENF, Campos dos Goytacazes, Rio de Janeiro - Brasil.

3 Engenheiro de Produção, Doutor em Ciência e Engenharia de Materiais, IFF-Centro, Campos dos Goytacazes, Rio de Janeiro - Brasil.

4 Engenheiro de Produção, Doutor em Ciência e Engenharia de Materiais, Pós-Doutorando, LAMAV, UENF, Campos dos Goytacazes, Rio de Janeiro - Brasil.

5 Engenheiro Mecânico, Doutor em Engenharia e ciência dos materiais, Professor, UENF, Campos dos Goytacazes, Rio de Janeiro - Brasil.

6 Engenheiro Metalúrgico, Doutor em Ciência e Engenharia de Materiais, Professor titular, IME, Rio de Janeiro, Rio de Janeiro - Brasil.

7 Engenheiro Mecânico, Doutor em Ciência e Engenharia de Materiais, Professor, ISECENSA, Campos dos Goytacazes, Rio de Janeiro - Brasil. 


\section{INTRODUÇÃO}

Atualmente, com o aumento da preocupação da sociedade com os impactos ambientais gerados por suas atividades, há cada vez mais estudos por materiais que minimizam ou que não sejam danosos ao meio ambiente [1].

Nesse novo contexto as fibras naturais, também chamadas de fibras lignocelulósicas, que já são utilizadas mantendo as propriedades em um nível bom para manter o produto competitivo, em áreas como a automotiva e a construção civil [1-5], inserem-se como uma boa alternativa para serem utilizadas como reforço de materiais compósitos poliméricos, substituindo as fibras sintéticas, nocivas ao meio ambiente, os compósitos reforçados com fibras naturais são chamados de biocompósitos. As fibras naturais, são biodegradáveis, renováveis, recicláveis, são facilmente descartados ou incinerados, e como são neutras em relação a emissão de $\mathrm{CO}_{2}$ não são nocivas ao meio ambiente, pois $\mathrm{o}^{\mathrm{CO}_{2}}$ é considerado hoje o principal responsável pelo aquecimento global, diferentemente das fibras sintéticas $[1,2,6,7]$. Além das vantagens ecológicas, as fibras lignocelulósicas possuem também vantagens técnicas em relação com as fibras sintéticas, como a leveza e o baixo custo, a relativa lisura em comparação com a fibra de vidro, gerando menos desgaste nos equipamentos [8].

O objetivo desse estudo é analisar a adesão das fibras de PALF no poliéster em função do valor do comprimento crítico, das fibras de PALF e a tensão cisalhante da interface fibra/matriz através de um ensaio de pullout.

\section{MATERIAIS E MÉTODOS}

O material utilizado nesse trabalho foi a fibra de PALF fornecido pela Desigan Fibras Naturais Fig. 1; e a resina foi do tipo poliéster com o endurecedor metil-etil-cetona em 0,5 vol\%.

Inicialmente 100 fibras de PALF foram aleatoriamente retiradas do pacote e 0 diâmetro equivalente correspondente a média de duas medidas da seção transversal da fibra em cinco pontos distintos, para cada fibra, foi medido em um projetor de perfil Nikon 6C.

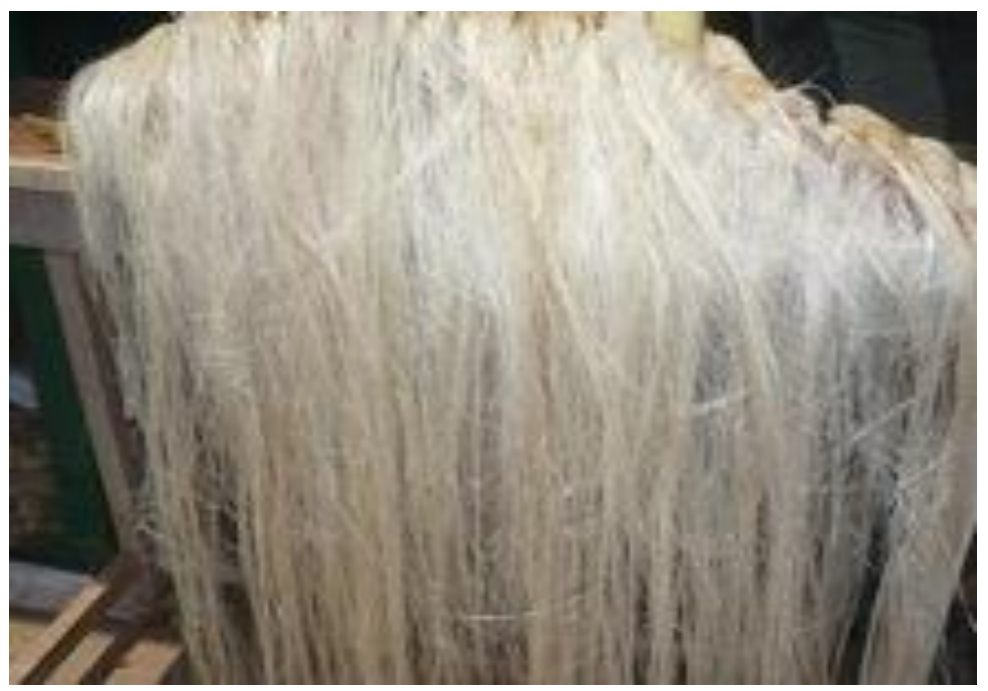

Figura 1. Um pequeno maço de fibras de PALF.

O histograma na Fig. 2 mostra uma grande dispersão nos valores do diâmetro da fibra de PALF, consequência da heterogeneidade das características físicas das 
fibras lignocelulósicas. O diâmetro varia entre 0,10-0,28 mm com um diâmetro médio de $0,20 \mathrm{~mm}$.

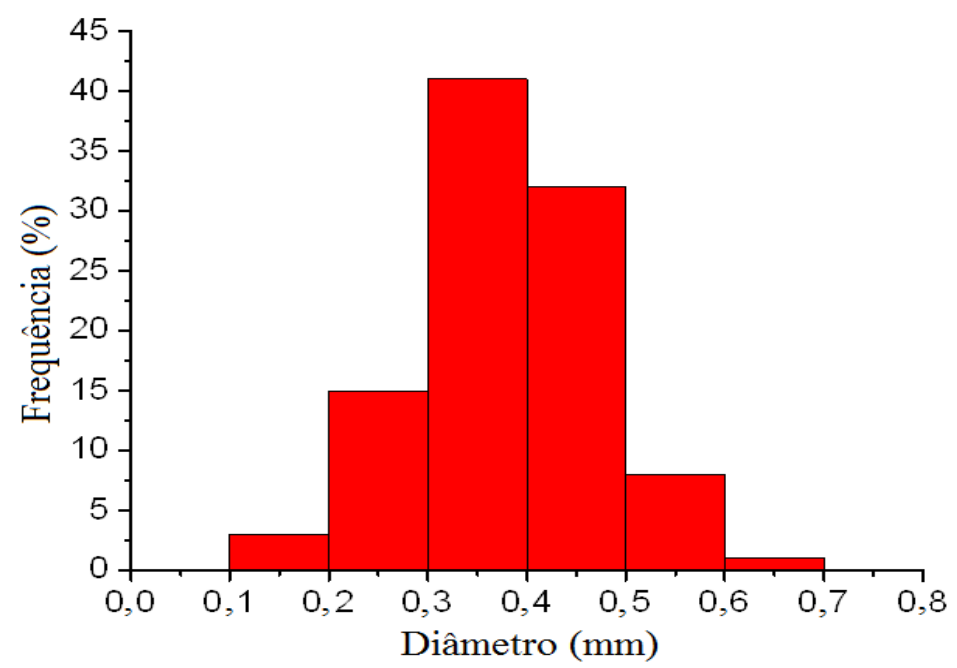

Figura 2. Histograma da distribuição do diâmetro das fibras de PALF.

Para a fabricação dos corpos de prova, para os ensaios mecânicos de pullout, utilizou-se um canudo de $6,5 \mathrm{~mm}$ de diâmetro como molde, onde dentro deste molde, uma fibra de PALF ficou misturada com a resina poliéster com o catalisador metil-etil-cetona, a diferentes profundidades: 0,$8 ; 1,1 ; 1,4 ; 1,7 ; 2 ; 2,3 \mathrm{~mm}$. Após a cura de $24 \mathrm{~h}$, o molde foi destruído, restando assim só o compósito.

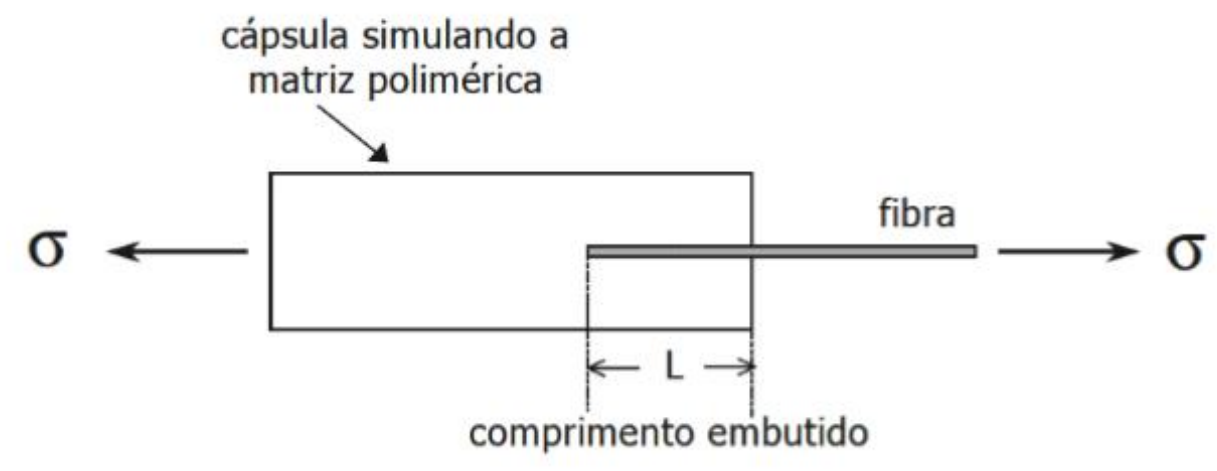

Figura 3. Esquema do corpo de prova utilizado no ensaio de pullout.

Os ensaios de pullout foram realizados em uma máquina universal de ensaios mecânicos, Instron 5582, com $100 \mathrm{kN}$ de capacidade. A velocidade do ensaio foi de $5 \mathrm{~mm} / \mathrm{min}$., temperatura de $22^{\circ} \mathrm{C}$ e pressão de $0,3 \mathrm{Mpa}$ na garra.

\section{RESULTADOS E DISCUSSÃO}

A Tabela 1 mostra os resultados obtidos no ensaio, as tensões médias necessárias para provocar o deslizamento da fibra de PALF com os devidos desvios padrões, com seu respectivo valor de embutimento. 
Tabela 1. Tensão média e desvio padrão para fibras de PALF em matriz de poliéster

\begin{tabular}{|c|c|}
\hline $\begin{array}{c}\text { Comprimento do } \\
\text { Embutimento (mm) }\end{array}$ & $\begin{array}{c}\text { Tensão Média e Desvio } \\
\text { Padrão (Mpa) }\end{array}$ \\
\hline 0,8 & $14,31 \pm 7,20$ \\
\hline 1,1 & $100,27 \pm 33,70$ \\
\hline 1,4 & $134,55 \pm 50,23$ \\
\hline 1,7 & $142,43 \pm 47,30$ \\
\hline 2,0 & $151,12 \pm 60,60$ \\
\hline 2,3 & $135,33 \pm 44,10$ \\
\hline
\end{tabular}

Como pode ser notado na Tabela 1 , o valor da tensão média aumenta à medida que, as fibras se encontram mais embutidas no poliéster. Apenas no embutimento de 2,3 $\mathrm{mm}$ isso não ocorre. Para 2,3 mm o valor de tensão média encontrado foi de 135,33 Mpa, valor inferior aos encontrados para 2,0 e 1,7 mm, 151,12 e 142,43 Mpa respectivamente.

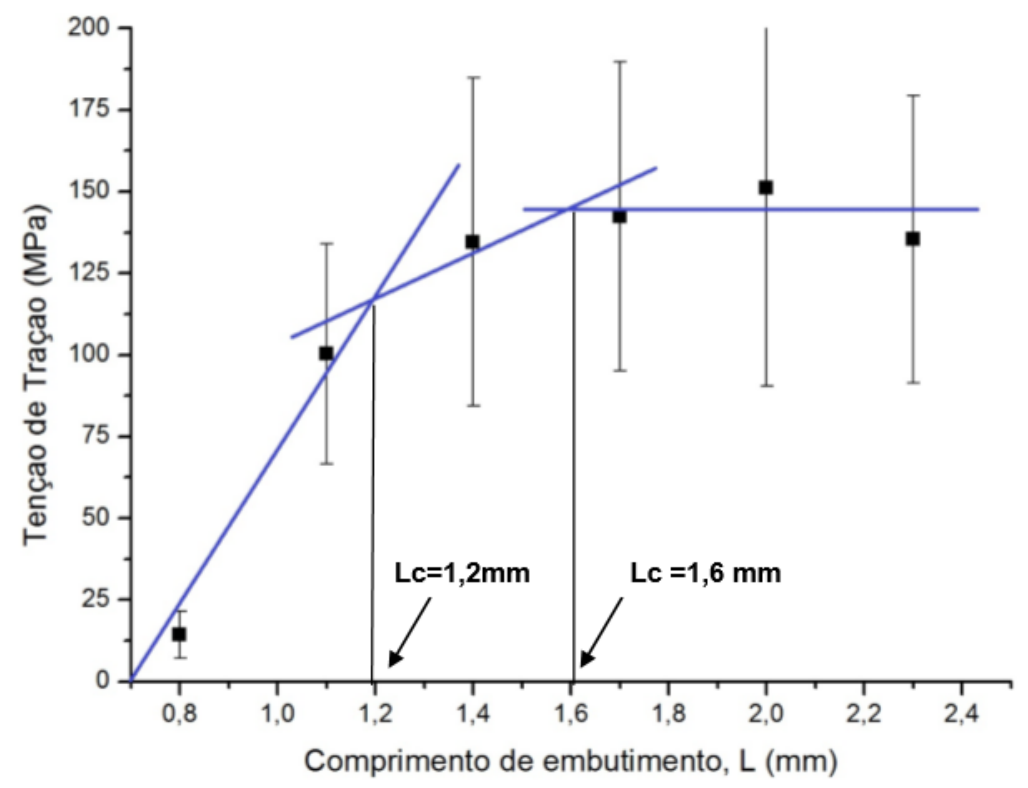

Figura 4. Tensão de tração e seu correspondente embutimento.

No gráfico apresentado na Fig. 4, a primeira reta, representa o ajuste linear para os três primeiros comprimentos de embutimento, 0,8, 1,1 e 1,4 $\mathrm{mm}$ respectivamente. A segunda para os comprimentos de embutimento de 1,1, 1,4 e 1,7 mm. E a terceira linha para os três últimos comprimentos de embutimento, 1,7, 2,0 e 2,3 mm.

$O$ valor de comprimento crítico de $L c=1,2 \mathrm{~mm}$, correspondente a interseção das duas primeiras retas dos ajustes lineares é o comprimento crítico limite encontrado para o livre desacoplamento da interface fibra/matriz. Já o segundo comprimento crítico, Lc $=1,6 \mathrm{~mm}$, interseção das duas últimas retas de ajuste linear é o valor de comprimento pelo qual não há mais o desacoplamento da fibra em relação a matriz.

Através do comprimento crítico achado, é possível calcular a tensão cisalhante interfacial, $\tau_{c}$, que mede a tensão cisalhante na interface fibra/matriz. Através da equação abaixo:

$$
\tau_{c}=\frac{d \sigma_{f}}{2 L_{c}}
$$


Sendo d, o diâmetro da fibra, $\sigma_{f}$, resistência a tração e $L_{c}$, o comprimento crítico.

Substituindo os valores de $\mathrm{L}_{c}=1,20 \mathrm{~mm}$ e $\mathrm{d}=0,20 \mathrm{~mm}$, e $\sigma_{f}=140 \mathrm{Mpa}$, obtém-se a tensão cisalhante na interface da fibra/matriz de 11,66 MPa.

\section{CONCLUSÃO}

- Os ensaios de pullout mostraram o comprimento crítico limite de $1,20 \mathrm{~mm}$ para a matriz de poliéster. Também mostraram a tensão cisalhante na interface fibra/matriz de 11,66 MPa.

\section{AGRADECIMENTOS}

Os autores agradecem o apoio a esta investigação pelas agências brasileiras: CNPq, CAPES e FAPERJ.

\section{REFERÊNCIAS}

[1] Wambua P, Ivens I, Verpoest I. Natural fibers: can they repalce glass and fibre reinforced plastics?. Composites Science and Technology, 63 (2003) 1259-1264.

[2] Zah R, Hischier R, Leão AL, Braun I. Curaua fibers in the automobile industry - A sustainability assessment. J. Cleaner Production, 15 (2007) 1032-1040.

[3] Satyanarayana KG, Guimarães JL, Wypych F. Studies on lignocellulosic fibers of Brazil. Part I: Source, production, morphology, properties and applications". Composites: Part A, 38, (2007) 1694-1709.

[4] Costa LL, Monteiro SN, Loiola RL. Mechanical behavior of polyester composites reinforced with continuous bamboo fibers. Proceedings of the Characterization of Minerals, Metals \& Materials - TMS Conference 2010 (Seattle, WA, USA, February 2010) 1-6.

[5] Monteiro SN, Pereira AC, Inácio WP. Izod impact energy of polyester matrix composites reinforced with aligned sisal fibers. In: Associação Brasileira de Metalurgia e Materiais - ABM, São Paulo, SP, Julho, 2011, p. 1-12.

[6] Mohanty AK, Misra M, Hinrichsen G. Biofibers, biodegradable polymers and biocomposites: an overview, Macromolecular Mat. And Engineering, 276/277 (2000), 1-24.

[7] Gore A. An Inconvenient Truth. The Planetary Emergency of Global Warming and What We Can do About It (Emmaus, Pennsylvania, USA: Rodale Press, 2006).

[8] Monteiro SN, Lopes FPD, Ferreira AS, Nascimento DCO. Natural fiber polymer matrix composites: cheaper, tougher and environmentally friendly. JOM, 61(1) (2009) 17-22. 